\title{
The phenotype of cancer cell invasion controlled by fibril diameter and pore size of $3 \mathrm{D}$ collagen networks
}

\author{
Jiranuwat Sapudom, ${ }^{a}$ Stefan Rubner, ${ }^{a}$ Steve Martin, ${ }^{a}$ Tony Kurth, ${ }^{b}$ Stefanie Riedel,,${ }^{b}$ \\ Claudia T. Mierke ${ }^{b}$ and Tilo Pompe ${ }^{a,{ }^{*}}$
}

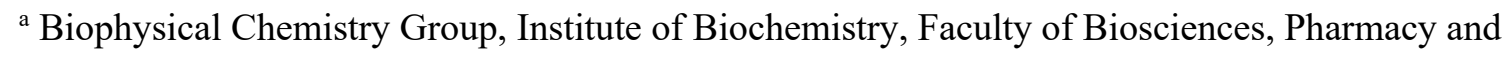
Psychology, Universität Leipzig, Leipzig 04103, Germany.

${ }^{\mathrm{b}}$ Biological Physics Division, Institute for Experimental Physics I, Faculty of Physics and Earth Science, Universität Leipzig, 04103 Leipzig, Germany.

* To whom Correspondence should be addressed

Tilo Pompe

Postal address:

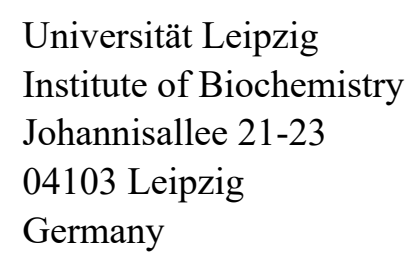

Telephone/Fax:

+493419736931/9

Email:

tilo.pompe@uni-leipzig.de

This article was published in final edited form as:

Sapudom J, Rubner S, Martin S, Kurth T, Riedel S, Mierke CT, Pompe T. The phenotype of cancer cell invasion controlled by fibril diameter and pore size of 3D collagen networks. Biomaterials 52:367-75 (2015). 


\begin{abstract}
The behavior of cancer cells is strongly influenced by the properties of extracellular microenvironments, including topology, mechanics and composition. As topological and mechanical properties of the extracellular matrix are hard to access and control for in-depth studies of underlying mechanisms in vivo, defined biomimetic in vitro models are needed. Herein we show, how pore size and fibril diameter of collagen I networks distinctively regulate cancer cell morphology and invasion. Three-dimensional collagen I matrices with a tight control of pore size, fibril diameter and stiffness were reconstituted by adjustment of concentration and $\mathrm{pH}$ value during matrix reconstitution. At first, a detailed analysis of topology and mechanics of matrices using confocal laser scanning microscopy, image analysis tools and force spectroscopy indicate pore size and not fibril diameter as the major determinant of matrix elasticity. Secondly, by using two different breast cancer cell lines (MDA-MB-231 and MCF-7), we demonstrate collagen fibril diameter - and not pore size - to primarily regulate cell morphology, cluster formation and invasion. Invasiveness increased and clustering decreased with increasing fibril diameter for both, the highly invasive MDA-MB-231 cells with mesenchymal migratory phenotype and the MCF-7 cells with amoeboid migratory phenotype. As this behavior was independent of overall pore size, matrix elasticity is shown to be not the major determinant of the cell characteristics. Our work emphasizes the complex relationship between structural-mechanical properties of the extracellular matrix and invasive behavior of cancer cells. It suggests a correlation of migratory and invasive phenotype of cancer cells in dependence on topological and mechanical features of the length scale of single fibrils and not on coarse-grained network properties.
\end{abstract}

Keywords: collagen; cell morphology; ECM (extracellular matrix); microstructure; mechanical properties; invasion 


\section{Introduction}

Leaving the primary tumor and invading the surrounding extracellular matrix $(\mathrm{ECM})$ is a key step in cancer cell invasion. Similarly important are steps in metastasis, when single tumor cell migration into healthy tissues at distant sites forms a new tumor in another microenvironment. Unfortunately these processes of cancer cell dissemination, invasion and metastasis are still not well understood [1-5]. Reasons for that may lie in the fast and complex alteration of cancer cells gene expression and biochemical signaling, but also in the changes to the biochemical composition and biophysical characteristics of the surrounding microenvironment by the cells.

Cancer cells have a distinctive ability to adapt to and communicate with their microenvironment. Hence, tumor-associated alterations in the extracellular microenvironment such as the composition, pore size and elasticity have a feedback onto the behavior of tumor cells themselves [6-10]. In this context it is known that the mechanical properties of tumors and tumor microenvironments change during tumor progression [9, 11-13]. Such mechanical changes of the surrounding ECM have been shown to trigger tumor cell behavior or even tumor-like behavior of non-tumorigenic cells [14]. Stiffening of tumor-associated ECM by deposition of collagen fibrils and increase of the fibril diameter are often observed in cancerous tissues suggesting a loss of control mechanisms in the regulation of collagen fibril formation [6].

However, a deep understanding of underlying mechanisms of the correlation between the mechanical properties and tumor formation as well as tumor progression is still hampered by the complexity of the involved signaling cascades, the heterogeneity of relevant cell populations and the shortcomings of model systems. While in vivo systems are difficult to investigate at high information depth, in vitro systems frequently lacking important biomimetic parameters of the in vivo situation like appropriate mechanics and dimensionality [15-18].

Due to that fact in vitro cell studies culture in 3D biomimetic matrices is a growing field as it offers a better representation of a natural cellular microenvironment in physiologically and pathologically relevant conditions. While synthetic systems frequently allow for well-defined preparation, they usually lack the complexity needed to address physiological questions. On the other hand naturally derived and reconstituted matrices are frequently hard to characterize and to reproduce in a welldefined manner [18]. In order to use matrices from naturally derived components good characterization of relevant parameters is a prerequisite for meaningful studies.

Type I collagen (Coll I) is a widely used ECM component due to its very high abundance in mammalian tissues. It is a gold standard for reconstituting 3D cell culture scaffolds regarding its fibrillar structure and ability to form networks of reasonable mechanical properties in vitro [19]. Coll I matrices facilitate cell attachment, proliferation and differentiation through receptor-mediated interactions predominantly via integrins [20-22]. Cell migration and invasion in 3D matrices are dynamical processes predominantly influenced by biophysical features of the surrounding matrix. Therefore, pore size and network elasticity are intensely discussed parameters to affect cell behavior $[13,23-26]$. However, less is known about how the Coll I fibril diameter specifically influences cell behavior, especially of cancer cells. In order to address such a question, 3D Coll I matrices with controlled fibril diameter are required. Recent reports emphasize the importance of such scaffolds as no direct correlation to matrix elasticity was found in contrast to previous reports [26, 27]. In vivo, fibroblasts regulate the diameter of Coll I fibrils by controlling type III and V collagen concentrations as nucleators during the process of Coll I fibrillogenesis [28]. In contrast, the fibril diameter can be regulated in vitro by temperature and $\mathrm{pH}$ during Coll I self-assembly, as well as by type $\mathrm{V}$ collagen concentration [24, 29-33]. Hence, there exist options for tuning topological features of Coll I matrices in vitro in order to generate well-defined 3D matrices. However, a comprehensive and independent modulation of those parameters and a correlated characterization of 3D Coll I matrices, i.e. pore size, fibril diameter and elasticity, is lacking. Hence, using well-defined 3D matrices with different 
topological and mechanical properties is envisioned to better clarify cancer cell behavior in a more physiological context.

\section{Materials and methods}

\subsection{Reconstitution of 3D Coll I matrices}

To immobilize 3D collagen matrices onto glass coverslips covalently, amine-functionalized glass coverslips of $13 \mathrm{~mm}$ diameter (VWR international, Leuven, Belgium) were coated with a thin layer of 0.14 wt.\% poly(styrene-alt-maleic anhydride) (PSMA; MW 30000 g/mol, Sigma-Aldrich, Steinheim, Germany) as published previously [34]. 3D collagen matrices were reconstituted using rat tail type I collagen (Coll I) (Corning, New York, USA). Final collagen concentrations of 2, 2.5 and $3 \mathrm{mg} / \mathrm{ml}$ were adjusted by mixing with $0.25 \mathrm{M}$ phosphate buffer to achieve final $\mathrm{pH}$ values of $7,7.5$ and 8 . Coll I solutions were prepared and kept on ice $\left(4{ }^{\circ} \mathrm{C}\right)$ to prevent polymerization for $60 \mathrm{~min}$. $\mathrm{pH}$ value of Coll I solutions was determined prior to network formation using universal indicator solution $\mathrm{pH}$ (Sigma-Aldrich) (see also Fig. S1) and pH electrode (Mettler-Toledo, Gießen, Germany). Subsequently, $30 \mu 1$ collagen solution was transferred onto PSMA-coated coverslips and polymerized at $37{ }^{\circ} \mathrm{C}, 5 \% \mathrm{CO}_{2}$ and $95 \%$ humidity for $90 \mathrm{~min}$. Afterwards that Coll I matrices were washed 3 times with phosphate buffered saline (PBS) (Biochrom, Berlin, Germany) and equilibrated at these neutral conditions. The reconstituted Coll I matrices, with a resulting thickness of about 250-300 $\mu \mathrm{m}$, were kept in a hydrated state prior to topological and mechanical characterization as well as cell culture studies.

\subsection{Kinetics of Coll I fibril formation}

For analysis of Coll I fibril formation kinetics, $100 \mu 1$ of prepared Coll I solution at different $\mathrm{pH}$ was transferred to a pre-chilled $\left(4{ }^{\circ} \mathrm{C}\right)$ 96-well microplate (Greiner, Bahlingen, Germany) prior to polymerization. 96-well microplates were loaded into a pre-warmed $\left(37^{\circ} \mathrm{C}\right)$ plate reader (TECAN Infinite F200 Pro, TECAN, Grödig, Austria) and turbidity at $405 \mathrm{~nm}$ was measured at $1 \mathrm{~min}$ intervals for $90 \mathrm{~min}$. Measurements were performed in three independent experiments.

\subsection{Topological characterization of 3D Coll I matrices}

3D Coll I matrices were stained with $50 \mu \mathrm{M}$ 5-(and-6)-Carboxytetramethylrhodamine succinimidyl ester (5(6)-TAMRA-SE) (Invitrogen, Carlsbad, USA) at room temperature for $1 \mathrm{~h}$ and rinsed 3 times with PBS. Collagen matrices were imaged with confocal laser scanning microscope LSM700 (Zeiss, Jena, Germany) using 40×/NA 1.3 oil immersion objective. Acquired images were 16-bit color depth, $1024 \times 1024$ pixels in resolution and a vertical stack size of 200 images (equivalent to $100 \mu \mathrm{m}$ ). The voxel size of the acquired images was $0.13 \times 0.13 \times 0.5 \mu \mathrm{m}(\mathrm{x} \times \mathrm{y} \times \mathrm{z})$. Representative sample images of the microarchitecture were generated from summed z-stacks of $50 \mu \mathrm{m}$ in depth using Imaris (Bitplane, Zurich, Switzerland). Pore size and fibril diameter were analyzed as described by Franke et.al. [23] using a home-built image processing procedure with erosion algorithm and autocorrelation method, respectively. This topology analysis was performed at least in triplicates with 6 positions per sample. Fibril length of 40 fibrils in total from 3 independent experiments was manually analyzed using the ImageJ fibril tracking tool (NIH, USA).

\subsection{Colloidal probe force spectroscopy of 3D Coll I matrices}

Elasticity of 3D Coll I matrices was determined using colloidal probe force spectroscopy (NanoWizard 3 AFM, JPK Instruments, Berlin, Germany). The colloidal probes were prepared by attaching a $50 \mu \mathrm{m}$ glass microbead (Polyscience Europe GmbH, Eppelheim, Germany) to a tipless MLCT rectangular cantilever ( $\mu$ Mash, Wetzlar, Germany) using an epoxy glue (Mettler-Toledo, Gießen, Germany). The spring constant of MLCT cantilever (approx. $60 \mathrm{nN} / \mathrm{m}$ ) was evaluated by the thermal noise method [35]. All experiments were performed in PBS buffer at room temperature. At least 50 force-distance curves were measured at 3 positions of each Coll I matrix with 3 independent 
experiments. The Young's modulus of the 3D collagen matrices was determined from the forcedistance curves by fitting the Hertz model with an indentation of 3 to $7 \mu \mathrm{m}$.

\subsection{Cell culture}

3D Coll I matrices were placed into 24 -well plate (Greiner, Bahlingen, Germany). $1 \times 10^{4}$ cells of MDA-MB-231 (human breast carcinoma cell line) and MCF-7 (human breast adenocarcinoma cell line) were seeded on top of 3D Coll I matrices and cultured in DMEM (Biochrom, Germany) supplemented with 10\% fetal calf serum (Biochrom, Germany) and 1\% ZellShield (Biochrom, Germany) at $37{ }^{\circ} \mathrm{C}, 5 \% \mathrm{CO}_{2}$ and $95 \%$ humidity.

Cell proliferation was determined after 4 days by means of commercial WST-1 assay (Roche, Germany). Cells were rinsed 3x with Hanks' balanced salt solution (HBSS, Sigma-Aldrich) with $\mathrm{Ca}^{2+}$ and $\mathrm{Mg}^{2+}$ and were subsequently incubated for $2 \mathrm{~h}$ with $500 \mu \mathrm{WST}-1$ solution (1:10 dilution with cell culture medium) at cell culture condition. Supernatants were collected and $100 \mu 1$ of each supernatant were transferred to 96-wells plates. The absorbance was measured at a wavelength of $450 \mathrm{~nm}$ with a multi-well plate reader.

\subsection{Analysis of cell invasion and morphology}

Cell invasion and morphology were analyzed after 4 days of culture. For analysis, cells were fixed in $4 \%$ paraformaldehyde (Roth, Karlsruhe, Germany) for $10 \mathrm{~min}$ at room temperature and rinsed 3 times with PBS. Afterwards, cells were permeabilized with 0.1\% Triton X100 (Roth, Germany) for 10 min at room temperature and rinsed 3 times with PBS. For analysis of cell invasion and morphology, cells were stained with DAPI (Invitrogen, Germany) and Alexa Fluor 488 Phalloidin (Invitrogen, Germany) for $30 \mathrm{~min}$ at room temperature and rinsed 3 times with PBS. Cells were imaged using an AxioObserver.Z1 with scanning stage (Zeiss, Jena, Germany) using a $10 \times$ objective. Images were $1388 \times 1040$ pixels in resolution with $5 \mu \mathrm{m}$ z-distance. At least 200 cells per experimental condition (3 positions from 3 independent experiments) were analyzed using DAPI signal from individual cell nuclei. Cells located $>10 \mu \mathrm{m}$ below the Coll I layer surface were counted as invasive cells. Cell morphology was imaged using Alexa Flour 488 Phalloidin signal. Aspect ratio (major axis/minor axis) was determined using ImageJ. Cells with an aspect ratio $<1.5$ were characterized as round cells. Cluster formation was manually counted, whereby cells without contact to other cells were counted as single cells (single object). For morphological and cluster formation analysis, at least 360 cells of 3 positions from 3 independent experiments were analyzed per experimental condition. For each cell invasion experiment, at least 200 cells of 3 positions from 3 independent experiments were analyzed per experimental condition. In addition, cells were imaged with 40×/NA 1.3 oil immersion oil objective using confocal laser scanning microscope LSM700 to receive high resolution images. Experiments were performed in 3 independent experiments.

\subsection{Data analysis and statistics}

Experiments were performed at least in triplicate, if not otherwise stated. Error bars indicate standard deviation (SD). Levels of statistical significance were determined using an unpaired t-test using OriginPro 8 (OriginLab Corp., USA). Significance level was set at 0.05 .

\section{Results and discussion}

The variation of Coll I concentration and $\mathrm{pH}$ condition during Coll I fibril formation are practical strategies to control parameters of reconstituted 3D Coll I matrices, as reported [23, 24, 32, 36]. However, a comprehensive picture of the independent variation of important topological parameters, their detailed characterization and correlation to mechanical matrix properties as well as cell behavior is still lacking. 


\subsection{Influence of self-assembly pH on Coll I fibrillogenesis}

We firstly examined the fibrillogenesis kinetics by turbidity measurements at $405 \mathrm{~nm}$ at $37^{\circ} \mathrm{C}$ and 1 min for $90 \mathrm{~min}$. Coll I concentration of $2,2.5$ and $3 \mathrm{mg} / \mathrm{ml}$ were prepared by mixing with phosphate buffer to achieve $\mathrm{pH}$ values of 7, 7.5 and 8. As shown in Fig. 1, collagen fibrillogenesis kinetics revealed a typical sigmoidal turbidity-time curve with characteristic lag, growth and plateau phases. The rate of fibrillogenesis and turbidity are increased with increasing $\mathrm{pH}$ at the same Coll I concentration. At the same $\mathrm{pH}$ condition the rate of fibrillogenesis as well as the final turbidity increased with increasing Coll I, as exemplarily shown at $\mathrm{pH} 7$ (Fig. 1B). The data correlated well with previous reports in terms of the impact of collagen concentration and $\mathrm{pH}[23,37,38]$.

A

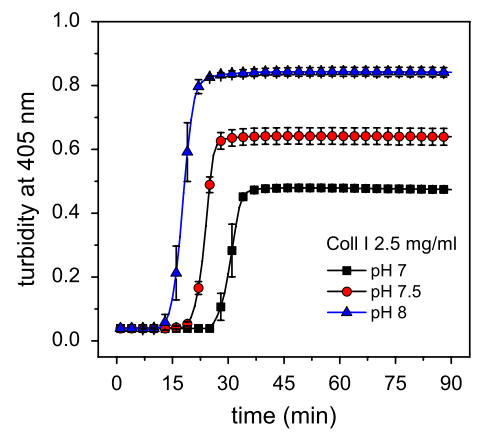

B

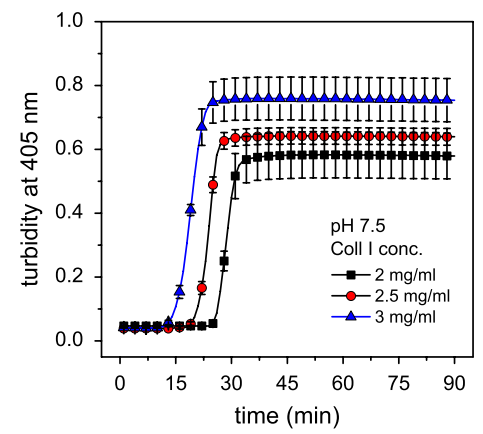

Figure 1. Kinetic studies of Coll I fibrillogenesis in dependence on $\mathrm{pH}$ and Coll I concentration. Increase in

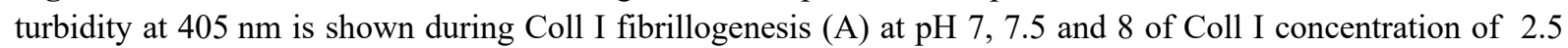
$\mathrm{mg} / \mathrm{ml}$ and (B) at $\mathrm{pH} 7.5$ with Coll I concentration of 2, 2.5 and $3 \mathrm{mg} / \mathrm{ml}$. Data are shown from 3 independent experiments with mean \pm SD.

\subsection{Topological and mechanical characterization}

Turbidity analysis provides insights into kinetics of the sol-gel transition, but provides no information regarding pore size and fibril morphology as well as mechanics. For that reason, we further performed topological and mechanical characterization to correlate conditions of Coll I reconstitution to biophysical properties of reconstituted 3D Coll I matrices, which are relevant for cell studies. The topology of 3D Coll I matrices was determined after labeling with TAMRA-SE and visualization with fluorescence cLSM using our home-built image analysis tools to automatically measure pore size and fibril diameter throughout the matrices. Fibril length was measured by ImageJ line tracking tool, while mechanics was determined using colloidal probe force spectroscopy.

Fig. 2 representatively illustrates the microarchitecture of Coll I matrices as a function of the Coll I concentration and the $\mathrm{pH}$ during fibrillogenesis. The cLSM images indicate that Coll I network density increases with Coll I concentration as expected. Collagen fibril diameter appears to be decreased with increasing $\mathrm{pH}$ and to be independent of Coll I concentration.

The quantitative topological analysis supports these visual impressions (Fig. 3). The fibril diameter is found to be thoroughly controlled by $\mathrm{pH}$ with 850,700 and $550 \mathrm{~nm}$ at $\mathrm{pH} 7,7.5$ and 8 , respectively and to be independent of Coll I concentration, see Fig. 3A. We further determined Coll I fibril length. As shown in Fig. 3B, Coll I fibril length is affected by $\mathrm{pH}$ with average lengths of 87, 110 and $41 \mu \mathrm{m}$ at $\mathrm{pH}$ 7, 7.5 and 8, respectively. However, fibril length appears to be not controlled by Coll I concentration. Remarkably, mean pore size of Coll I networks showed a different trend compared to fibril diameter. Pore size decreased with increasing Coll I concentration, but was only slightly affected by $\mathrm{pH}$. A slight decrease was observed when comparing $\mathrm{pH} 7$ and 7.5 conditions. However, no difference could be found when comparing $\mathrm{pH} 7$ and $\mathrm{pH} 8$ conditions (Fig. 3C). Mean pore sizes were found approx. $13 \mu \mathrm{m}, 9 \mu \mathrm{m}$ and $7 \mu \mathrm{m}$ for concentrations $2,2.5$ and $3 \mathrm{mg} / \mathrm{ml}$, respectively, which nicely resemble in vivo situation of collagen matrices in interstitial tissues [39]. When looking on pore size distributions (Fig. S2), we found further indication of altered fibril formation at high $\mathrm{pH}(\mathrm{pH} 8)$. 
While small and large pore sizes are similarly distributed for $\mathrm{pH} 7$ and 7.5 , there is a clear shift to an increased amount of smaller pores for $\mathrm{pH} 8$, in line with altered trend of fibril length at this $\mathrm{pH}$. An additional analysis indicated that pore size and fibril diameter were constant throughout the whole Coll I layer (Fig. S3, S4).

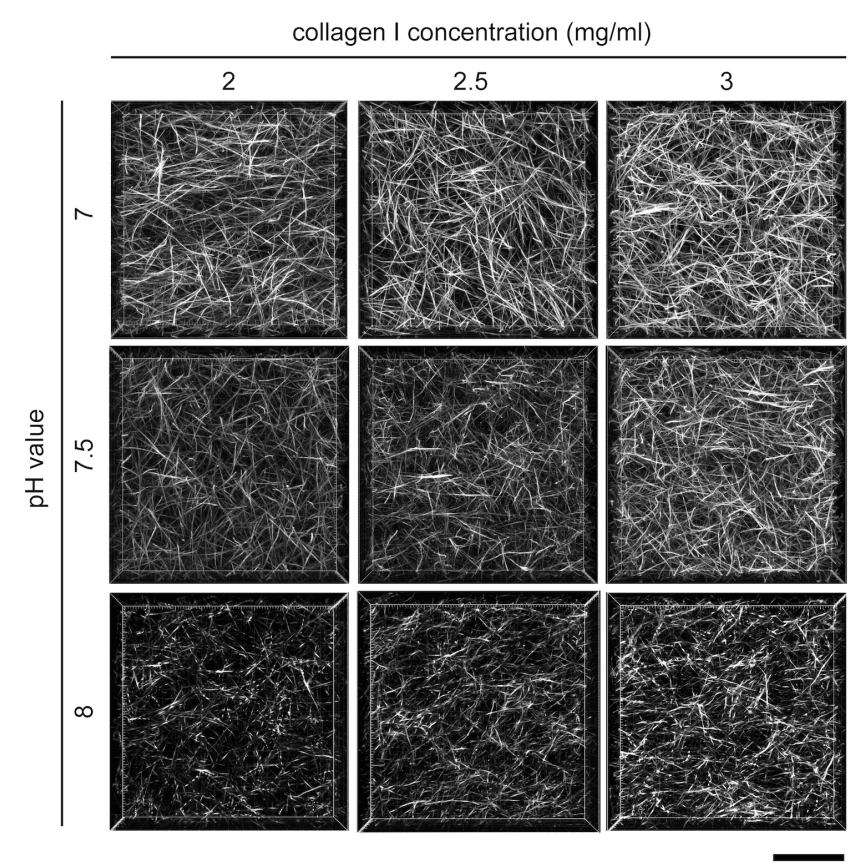

Figure 2. Microarchitecture of 3D Coll I matrices in dependence on $\mathrm{pH}$ and Coll I concentration. Representative cLSM images of Coll I matrices, stained with TAMRA-SE, are shown for Coll I concentrations of 2, 2.5 and 3 $\mathrm{mg} / \mathrm{ml}$ and $\mathrm{pH}$ of 7, 7.5 and 8. Images show summed z-stacks of $50 \mu \mathrm{m}$ depth. (Scale bar: $50 \mu \mathrm{m}$ ).

The mechanical properties of Coll I matrices are strongly influenced by topological parameters. We therefore analyzed elasticity of our matrices using colloidal probe force spectroscopy. We found an increasing elastic modulus with increasing Coll I concentration, namely 35, 50, $65 \mathrm{~Pa}$ for 2, 2.5 and 3 $\mathrm{mg} / \mathrm{ml}$ Coll I concentration reconstituted at $\mathrm{pH} 7$, respectively (Fig. 3D). However, the $\mathrm{pH}$ dependence gave another trend with a slight increase comparing $\mathrm{pH} 7$ and $\mathrm{pH} 7.5$ and almost no change comparing $\mathrm{pH} 7$ and $\mathrm{pH} 8$ for the same Coll I concentrations, e.g. 50, 90, $50 \mathrm{~Pa}$ for the $\mathrm{pH} 7,7.5$ and 8 at $2,5 \mathrm{mg} / \mathrm{ml}$ Coll I, respectively).

In general our results agree with other reports that increasing $\mathrm{pH}$ correlates with a decreasing fibril diameter $[31,32,40]$. It was argued that the net positive charges of Coll I are decreased at higher $\mathrm{pH}$ during fibril formation, resulting in increased hydrogen bonds between triple helix. This should finally lead to weaker lateral association into fibril because of the increase triple helix stability [30]. Furthermore, a change of fibril formation mechanism at very high $\mathrm{pH}$ was also reported by Raub et al. although at somewhat higher $\mathrm{pH}(>\mathrm{pH}$ 8.5) [32], which might be attributed to buffer conditions (phosphate buffer in our case) and Coll I source. Mertz et al. demonstrated that inorganic monovalent and divalent phosphates do not interact with Coll I monomers in solution. Therefore no enhanced Coll I assembly and stability can be observed [41]. In our case it leads to a change in pore size distribution (more smaller pores), decreased fibril length and decreased Young's modulus. 
A

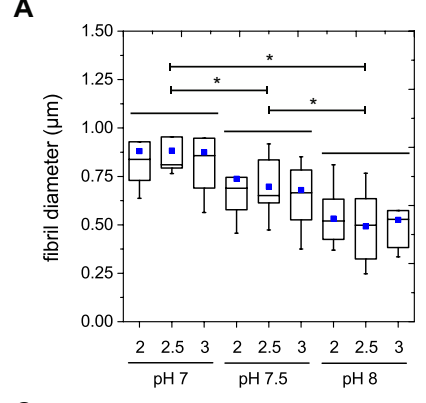

C

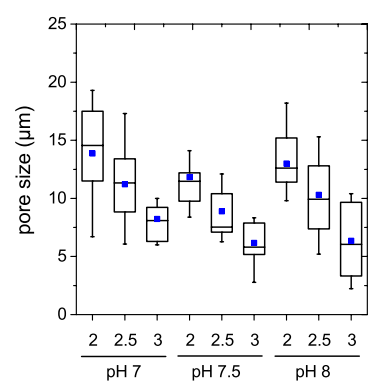

B
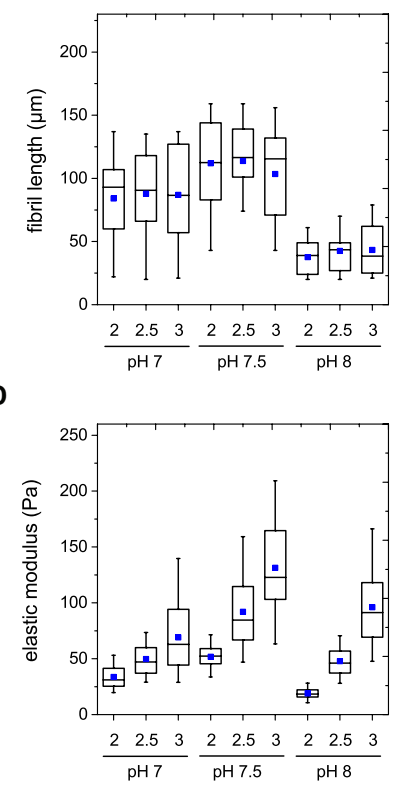

Figure 3. Topological and mechanical characteristics of reconstituted 3D Coll I matrices. (A) Fibril diameter, (B) fibril length, (C) pore size and (D) Young's modulus are shown in dependence on $\mathrm{pH}$ and Coll I concentration as determined by topology analysis from cLSM images and by colloidal probe force spectroscopy, respectively. Analysis of pore size and fibril diameter were performed at least in triplicates with 6 positions per sample. Fibril length was manually analyzed of 40 fibrils in total from 3 independent experiments. For the measurement of Young's modulus, at least 50 force-distance curves were measured at 3 positions for each Coll I matrices with 3 independent experiments. Data are represented as mean $\pm \mathrm{SD}$; * significance level of $\mathrm{p}<0.05$.

Our study adds more details on the correlation of Coll I matrix topology and mechanical properties as we are able to independently vary and correlate all parameters. We also believe that our approach for topology analysis provides a more reliable and meaningful measure as we explore the full thickness of Coll I matrices with 3D imaging by fluorescence cLSM and use an unbiased automatic measurement of fibril diameter and mean pore size including access to pore size distributions in contrast to previous reports using only single plane measurements, reflection mode imaging or manual measurements. Based on that, our study finds a direct correlation of mean pore size with Young's modulus of the Coll I matrices. Furthermore, Young's modulus is found to be independent of fibril diameter. This suggests the mean pore size, defined as the feature diameter at $50 \%$ of eroded pore area (see [23]), as a very meaningful topological measure of matrix elasticity. Fibril diameter might also influence the elastic properties of Coll I networks due to the increase of single fibril bending modulus with fibril thickness. However, we do not observe such a relationship. This observation is reasonable in the light of models on fibrillar networks of semi-flexible polymers suggesting interconnectivity as well as entanglement as major determinants of elastic network properties [42].

In summary, the topological and mechanical analysis of Coll I matrices in dependence on Coll I concentration and $\mathrm{pH}$ condition during fibrillogenesis underlines those parameters as powerful engineering tools to modulate Coll I matrices. By that approach 3D Coll I matrices can be produced with defined pore size, fibril diameter and correlated mechanical properties for in vitro cell culture studies.

\subsection{Impact of Coll I matrix topology and mechanics on cancer cell morphology and invasiveness}

The 3D Coll I matrices were further used to probe the impact of different cell microenvironments in terms of ECM topology and elasticity. While pore size and elasticity of 3D Coll I matrices were already used to study their influence on cancer cell invasion, our study distinctively determined cancer cell behavior as a function of Coll I fibril diameter while controlling the other parameters (pore size 
and elasticity) at the same time. Based on the characterization presented above, we chose one set of samples with constant pore size $(11 \mu \mathrm{m})$ and elasticity $(50 \mathrm{~Pa})$ and varied fibril diameter with $850 \mathrm{~nm}$, $700 \mathrm{~nm}$ and $550 \mathrm{~nm}$ using Coll I matrices reconstituted with $2.5 \mathrm{mg} / \mathrm{ml}$ at pH 7,2 $\mathrm{mg} / \mathrm{ml}$ at pH 7.5 and $2.5 \mathrm{mg} / \mathrm{ml}$ at $\mathrm{pH} 8$, respectively. An additional set of samples was chosen with same fibril diameter distribution, but decreased pore size, namely $7.5 \mu \mathrm{m}$ or $5.5 \mu \mathrm{m}$. For cell studies two breast cancer cell lines have been chosen with distinctively different migratory characteristics, which are used as standard tools in assessing biological and biophysical behavior of tumor cells [43, 44]. The human breast carcinoma cell line MDA-MB-231 exhibits a mesenchymal migration type, while the human breast adenocarcinoma cell line MCF-7 is characterized by an amoeboid migratory phenotype. Suspended cells were seeded on top of the Coll I matrices and cultured for 4 days. They were subsequently analyzed for morphology and invasion characteristics. To exclude an impact of differences in cell proliferation, it was determined at day 4 of cell culture and found to be not significantly different for the different matrices (Fig. S6).

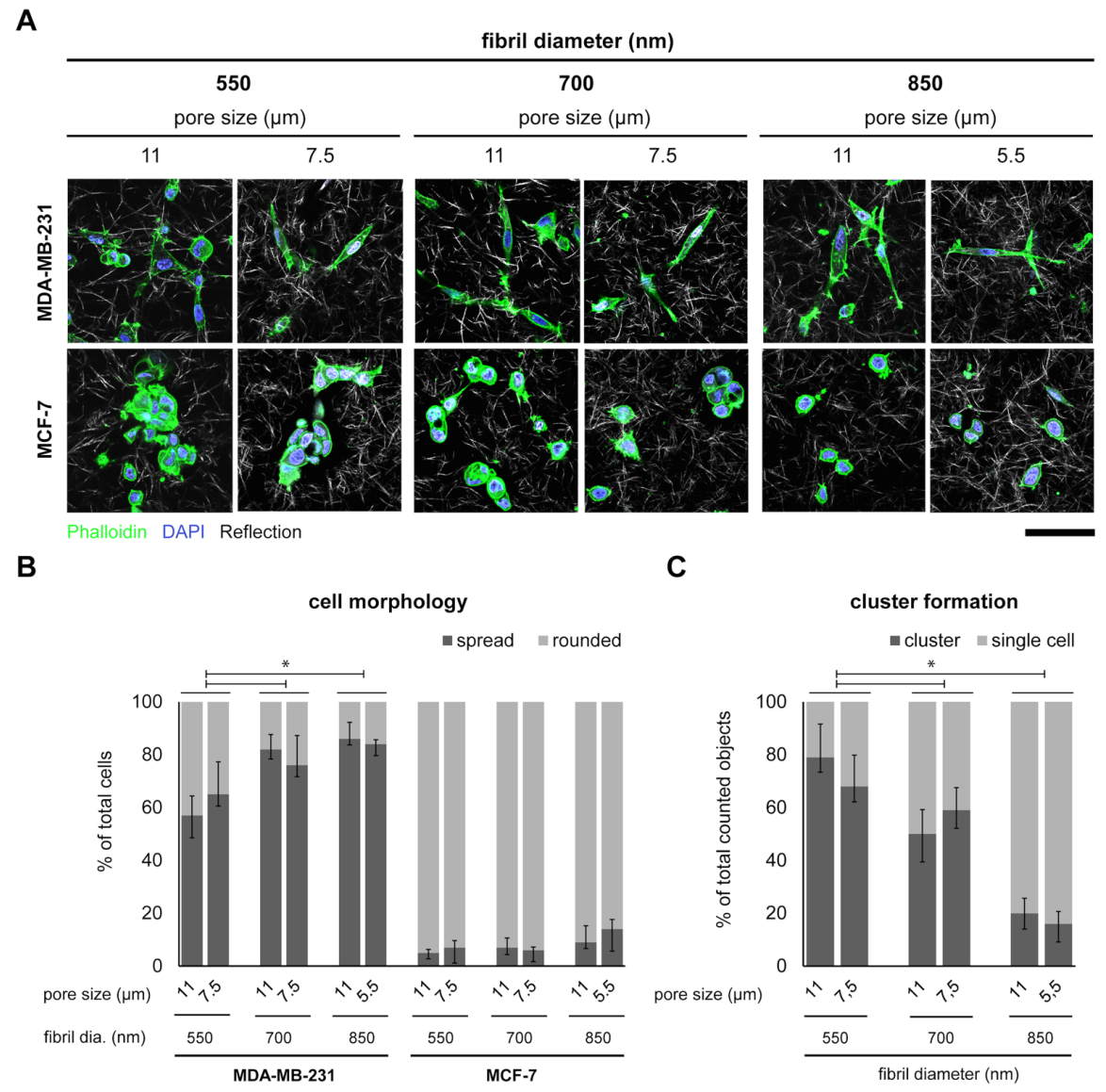

Figure 4. Impact of Coll I fibril diameter and pore size on cell morphology and cluster formation. (A) cLSM images of MDA-MB-231 and MCF-7 breast cancer cells cultivated on 3D Coll I matrices with fibril diameter of 550,700 and $850 \mathrm{~nm}$ and pore size of $11 \mu \mathrm{m}$ and 7.5/5.5 $\mu \mathrm{m}$ for 4 days. Images show nuclei (blue), actin filaments (green) and Coll I fibrils (white). (Scale bar: $50 \mu \mathrm{m}$ ), (B) Quantitative analysis of cell morphology of MDA-MB-231 and MCF-7 cells as a function of fibril diameter and pore size. Cells with a width-to-length ratio $>1.5$ were counted as spread cells (dark gray bar), while otherwise counted as round (light gray bar). (C) Quantitative analysis of cluster formation of MCF-7 cells, whereby cells without contact to other cells were counted as single cells (light gray bar). At least 360 cells of 3 positions from 3 independent experiments were analyzed per experimental condition. Significance was tested between samples of different fibril diameter and similar pore size. (Data represents as mean $\pm \mathrm{SD} ;{ }^{*}$ - significance level of $\mathrm{p}<0.05$ ). 
As shown in Fig. 4A, MDA-MB-231 cells appeared to change their shape to a more stretched form with increasing Coll I fibril diameter, whereas MCF-7 cells exhibit round morphology and did not change their morphology with increasing fibril diameter. Quantitative analysis of cell morphology showed cell number with spread cell morphology of MDA-MB-231 cells to significantly increase with increasing fibril diameter. In contrast, MCF-7 cells showed a constant rounded morphology in all matrices (Fig. 4B). MCF-7 cells are known to form clusters in 2D cell culture, which we also observed in 3D Coll I matrices with thin Coll I fibrils. However, cluster size and number of clusters was found to decrease with increasing Coll I fibril diameter (Fig. 4A, C and S5).

To verify whether these effects are influenced by fibril diameter only, we used a second set of 3D Coll I matrices with same fibril diameter distribution but smaller pore size and correlated higher Young's modulus. Interestingly, at decreased pore size (higher Young's modulus) we found likewise changes in cell morphology and cluster formation in dependence on fibril diameter, see Fig. 4A,C. The quantitative comparison indicated no significant differences in dependence on pore size (elasticity). Only a very slight increase in cell elongation of MDA-MB-231 cells could be observed for the stiffer matrices, which is typical for mesenchymal cells due to higher tensile forces applied in stiffer matrices.

A

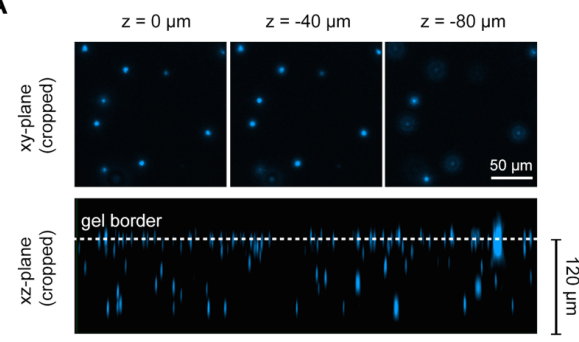

C

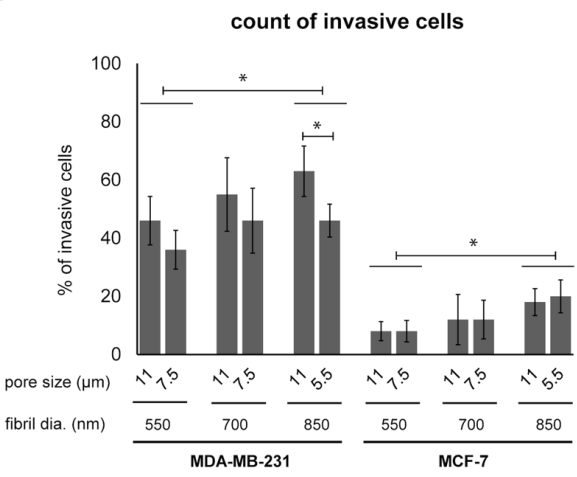

B

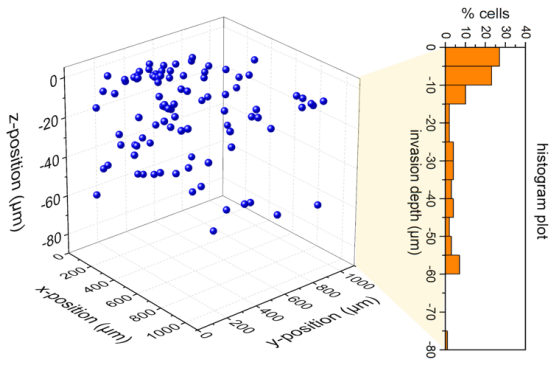

D

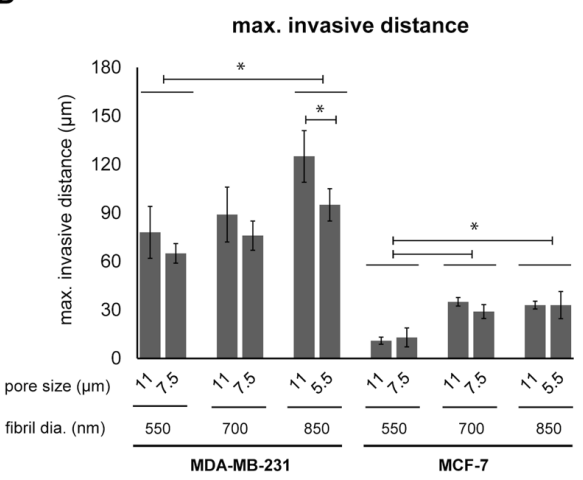

Figure 5. Invasion of cancer cells in dependence on Coll I fibril diameter and pore size after 4 days. (A) Typical images of invasion analysis using nuclei staining and fluorescence microscopy showing xy-plane at different depth of Coll I matrices and z-plane of stacked images. (B) Exemplary 3D graph and distribution in z-direction of invaded MDA-MB-231 cells cultivated on Coll I matrices with fibril size of $550 \mathrm{~nm}$ and pore size of $11 \mu \mathrm{m}$. (C) Quantitative analysis of number of invasive cells and (D) maximal invasive distance of MDA-MB-231 and MCF-7 cells are shown in dependence on fibril diameter and pore size. Cells found $>10 \mu \mathrm{m}$ beneath the Coll I matrix surface were counted as invasive cells. At least 200 cells of 3 positions from 3 independent experiments were analyzed per experimental condition. Significance was tested between samples of different fibril diameter and similar pore size. (Data are represented as mean $\pm \mathrm{SD} ;{ }^{*}$ - significance level of $\mathrm{p}<0.05$ ).

Hence, we can conclude that both cell lines, MDA-MB-231 and MCF-7, exhibit typical morphologies in respect to mesenchymal and amoeboid migratory phenotypes, and distinctively react to changes in fibril diameter of Coll I matrices, but not to pore size and matrix elasticity. This findings correlate well with recent reports stating similar independence of cell behavior on matrix elasticity [27]. This finding 
suggests small-scale mechanical properties of single fibrils, and not the large-scale network elasticity, as the major determinant of cell behavior in respect to morphology and clustering.

We further analyzed the invasion capability of both cells as a function of fibril diameter. Cell invasion was measured as the number of cells found $>10 \mu \mathrm{m}$ beneath the Coll I matrix surface after 4 days of cell culture. Fig. 5A shows a typical image from fluorescence microscopy of DAPI-stained MDA-MB231 cells visualizing xyz-positions of cells in a Coll I matrix reconstituted at $2.5 \mathrm{mg} / \mathrm{ml} \mathrm{Coll} \mathrm{I} \mathrm{and} \mathrm{pH}$ 8. Fig. 5B provides a 3D representation and a histogram of the distribution in z-direction. These data indicate distributed cells in xy-direction and half of cells invaded in the matrix $>10 \mu \mathrm{m}$. The highly invasive MDA-MB-231 cells were found to be dependent in invasion characteristics on fibril diameter and pore size with an increasing number of invasive cells in case of an increase in fibril diameter and an increase in pore size. Both trends were independent of each other. For the weakly invasive MCF-7 cells a similar dependence on fibril diameter was found, but to a much smaller extent. For MDA-MB231 around $50 \%$ were found to invade the matrices, while only $10 \%$ were determined for MCF-7 cells. Notably, no dependence was observed in dependence on pore size for the MCF-7 cells. The analysis of maximum invasion distance provided similar trends as for the number of invaded cells in respect to fibril diameter and pore size, see Fig. 5D.

Obviously there is a distinct correlation of migratory phenotype and invasion characteristics in dependence on distinct topological and mechanical parameters of the matrices. While invasion of MDA-MB-231 cells with a mesenchymal, highly invasive phenotype depended on fibril diameter and pore size, no pore size dependence is found for amoeboid, less invasive MCF-7 cells. This finding indicates small-scale fibril mechanics (bending stiffness of fibrils) to affect both mesenchymal and amoeboid cell migration and invasion, pore size and the correlated large-scale network elasticity to influence mesenchymal migration only.

Overall the results of the cell culture studies on the Coll I matrices indicate a distinctive regulation of morphological and invasive characteristics in dependence of specific parameters of Coll I matrix and the particular migratory phenotype of cancer cells. In agreement to literature we find a dependence on topology and mechanics of the matrices, however, we are additionally able to separate the impact of different features of different length scale by the usage of a comprehensive set of well-defined and tuned matrices. A transition from a rounded to an elongated morphology is found for highly invasive MDA-MB-231 cells in dependence on fibril diameter independent on pore size and Young's modulus. Hence, the mechanical properties of single fibril, like bending stiffness, seems to trigger cell behavior, which suggest this property of micrometer length scale to trigger the change of cell phenotype, and not network elasticity on the length scale of some tens of micrometer. Such a length scale compares well with the size of typical adhesion sites of cells, which are known to regulate cell mechanotransduction [45]. Similarly it is interesting to compare such a morphological transition to the phenomenon of mesenchymal-amoeboid transition (MAT) which is known from cancer cell migration by weakening of integrin-ECM interactions [46]. Our cell-matrix system might provide a relevant platform to study those phenomena.

On the other hand we found less invasive MCF-7 cells, characterized by an amoeboid migration, to show a decreased cluster formation with increasing fibril diameter, but again independent of pore size and Young's modulus. This observation is reminiscent of dissemination of cancer cells from a growing tumor, indicating that changes in matrix topology and mechanics and specifically thickness of fibrillar components could trigger invasion into healthy tissues by local changes of matrix topology and mechanics.

The common observation for both cell lines of an increased invasion with increasing fibril diameter correlates to the phenomenon of contact guidance [13,47]. In this context it is discussed that mechanosensory integrins [45] and Rho/ROCK-mediated directional contraction [48] lead to a directed migration of cells in dependence on the size and mechanical properties of one-dimensional objects, such as fibrils. Thicker fibrils could promote stronger cell-matrix interactions resulting in 
enhanced polarization of mesenchymal MDA-MB-231 evoked by higher forces and less clustering for MCF-7 cells due to stronger cell-matrix interactions than cell-cell interactions.

It is important to note, that our findings suggest that besides contact guidance phenomena the mechanical properties of single fibrils dominate the cell behavior despite the fact that overall matrix mechanics, Young's modulus, is correlated to pore size and not fibril diameter. Hence, we do not find a correlation of matrix stiffness and invasiveness as frequently reported in literature [14, 49, 50]. However, a recent report of no direct correlation of matrix elasticity and cell behavior of tumor cells supports our observation [27]. Even more, MDA-MB-231 cells invade the matrices to a smaller degree with an increasing Young's modulus. Probably the higher network density triggers not only stronger cell-matrix adhesions with higher forces and stronger polarization it obviously also hinders cells in migrating through the network correlating to known limitations in nuclei deformation at small pore sizes [39]. For the amoeboid migrating MCF-7 this mechanisms seems to be not important as they are suggested to squeeze through the network pores by pushing force rather pulling forces [46].

\section{Conclusion}

In conclusion, we generated 3D Coll I matrices with defined fibril diameter, pore size and Young's modulus by varying $\mathrm{pH}$ and Coll I concentration. We demonstrated that mean pore size determined by the topological analysis predominantly influences the Young's modulus of Coll I matrices, independent of fibril diameter. The topological and mechanical parameters of the Coll I matrices were further shown to lead to characteristic behavior of two different cancer cell lines in vitro. Invasion and cluster formation were demonstrated to be distinctively affected by the small-scale mechanics of Coll I fibrils and large-scale network pore size and mechanics in dependence on mesenchymal and amoeboid cell phenotype. These finding emphasizes the impact of the different hierarchical levels of ECM structures affecting cancer cell behavior in terms of invasion and metastasis. They also underpin the importance to use well-defined biomimetic 3D ECM matrices as in vitro models in further studies addressing clinically more relevant parameters of tumorbiology.

\section{Acknowledgement}

The authors acknowledge the support of grants from ESF 'European Social Funds' and Free State of Saxony (SAB 100147954, SAB 100148830) and from DFG INST 268/293-1 FUGG. Furthermore, we thank Ilja Iwlew for preparation of coated cover slips and Michael Ansorge for proofreading of the manuscript.

\section{References}

[1] Friedl P, Wolf K. Tumour-cell invasion and migration: diversity and escape mechanisms. Nat Rev Cancer. 2003;3:362-74.

[2] Braun S, Naume B. Circulating and disseminated tumor cells. J Clin Oncol. 2005;23:1623-6.

[3] Bacac M, Stamenkovic I. Metastatic cancer cell. Annu Rev Pathol. 2008;3:221-47.

[4] Rocken M. Early tumor dissemination, but late metastasis: insights into tumor dormancy. J Clin Invest. 2010;120:1800-3.

[5] Friedl P, Alexander S. Cancer invasion and the microenvironment: plasticity and reciprocity. Cell. 2011;147:992-1009.

[6] Brabek J, Mierke CT, Rosel D, Vesely P, Fabry B. The role of the tissue microenvironment in the regulation of cancer cell motility and invasion. Cell Commun Signal. 2010;8:22.

[7] Lu P, Weaver VM, Werb Z. The extracellular matrix: a dynamic niche in cancer progression. J Cell Biol. 2012;196:395-406.

[8] Levental KR, Yu H, Kass L, Lakins JN, Egeblad M, Erler JT, et al. Matrix crosslinking forces tumor progression by enhancing integrin signaling. Cell. 2009;139:891-906. 
[9] Baker EL, Lu J, Yu D, Bonnecaze RT, Zaman MH. Cancer cell stiffness: integrated roles of threedimensional matrix stiffness and transforming potential. Biophys J. 2010;99:2048-57.

[10] Mierke CT, Frey B, Fellner M, Herrmann M, Fabry B. Integrin alpha5betal facilitates cancer cell invasion through enhanced contractile forces. J Cell Sci. 2011;124:369-83.

[11] Kraning-Rush CM, Califano JP, Reinhart-King CA. Cellular traction stresses increase with increasing metastatic potential. PLoS One. 2012;7:e32572.

[12] Katira P, Zaman MH, Bonnecaze RT. How Changes in Cell Mechanical Properties Induce Cancerous Behavior. Physical Review Letters. 2012;108.

[13] Seewaldt V. ECM stiffness paves the way for tumor cells. Nat Med. 2014;20:332-3.

[14] Paszek MJ, Zahir N, Johnson KR, Lakins JN, Rozenberg GI, Gefen A, et al. Tensional homeostasis and the malignant phenotype. Cancer Cell. 2005;8:241-54.

[15] Griffith LG, Swartz MA. Capturing complex 3D tissue physiology in vitro. Nat Rev Mol Cell Biol. 2006;7:211-24.

[16] Infanger DW, Lynch ME, Fischbach C. Engineered culture models for studies of tumor-microenvironment interactions. Annu Rev Biomed Eng. 2013;15:29-53.

[17] Even-Ram S, Yamada KM. Cell migration in 3D matrix. Curr Opin Cell Biol. 2005;17:524-32.

[18] Nyga A, Cheema U, Loizidou M. 3D tumour models: novel in vitro approaches to cancer studies. J Cell Commun Signal. 2011;5:239-48.

[19] Stamov DR, Pompe T. Structure and function of ECM-inspired composite collagen type I scaffolds. Soft Matter. 2012;8:10200.

[20] Jokinen J, Dadu E, Nykvist P, Kapyla J, White DJ, Ivaska J, et al. Integrin-mediated cell adhesion to type I collagen fibrils. J Biol Chem. 2004;279:31956-63.

[21] Popov C, Radic T, Haasters F, Prall WC, Aszodi A, Gullberg D, et al. Integrins alpha2beta1 and alpha1lbeta1 regulate the survival of mesenchymal stem cells on collagen I. Cell Death Dis. 2011;2:e186.

[22] Carlson MA, Smith LM, Cordes CM, Chao J, Eudy JD. Attachment-regulated signaling networks in the fibroblast-populated 3D collagen matrix. Sci Rep. 2013;3:1880.

[23] Franke K, Sapudom J, Kalbitzer L, Anderegg U, Pompe T. Topologically defined composites of collagen types I and V as in vitro cell culture scaffolds. Acta Biomater. 2014;10:2693-702.

[24] Wolf K, Te Lindert M, Krause M, Alexander S, Te Riet J, Willis AL, et al. Physical limits of cell migration: control by ECM space and nuclear deformation and tuning by proteolysis and traction force. $\mathrm{J}$ Cell Biol. 2013;201:1069-84.

[25] Harjanto D, Maffei JS, Zaman MH. Quantitative analysis of the effect of cancer invasiveness and collagen concentration on 3D matrix remodeling. PLoS One. 2011;6:e24891.

[26] Friedl P, Sahai E, Weiss S, Yamada KM. New dimensions in cell migration. Nat Rev Mol Cell Biol. 2012;13:743-7.

[27] Guzman A, Ziperstein MJ, Kaufman LJ. The effect of fibrillar matrix architecture on tumor cell invasion of physically challenging environments. Biomaterials. 2014;35:6954-63.

[28] Sun M, Chen S, Adams SM, Florer JB, Liu H, Kao WW, et al. Collagen V is a dominant regulator of collagen fibrillogenesis: dysfunctional regulation of structure and function in a corneal-stroma-specific Col5a1null mouse model. J Cell Sci. 2011;124:4096-105.

[29] Doyle AD, Wang FW, Matsumoto K, Yamada KM. One-dimensional topography underlies threedimensional fibrillar cell migration. J Cell Biol. 2009;184:481-90.

[30] Leikin S, Rau DC, Parsegian VA. Temperature-favoured assembly of collagen is driven by hydrophilic not hydrophobic interactions. Nature Structural Biology. 1995;2:205-10.

[31] Harris JR, Soliakov A, Lewis RJ. In vitro fibrillogenesis of collagen type I in varying ionic and $\mathrm{pH}$ conditions. Micron. 2013;49:60-8.

[32] Raub CB, Unruh J, Suresh V, Krasieva T, Lindmo T, Gratton E, et al. Image correlation spectroscopy of multiphoton images correlates with collagen mechanical properties. Biophys J. 2008;94:2361-73.

[33] Raub CB, Suresh V, Krasieva T, Lyubovitsky J, Mih JD, Putnam AJ, et al. Noninvasive assessment of collagen gel microstructure and mechanics using multiphoton microscopy. Biophys J. 2007;92:2212-22.

[34] Pompe T, Zschoche S, Herold N, Salchert K, Gouzy MF, Sperling C, et al. Maleic anhydride copolymers--a versatile platform for molecular biosurface engineering. Biomacromolecules. 2003;4:1072-9.

[35] Hutter JL, Bechhoefer J. Calibration of atomic-force microscope tips. Review of Scientific Instruments. 1993;64:1868.

[36] Carey SP, Kraning-Rush CM, Williams RM, Reinhart-King CA. Biophysical control of invasive tumor cell behavior by extracellular matrix microarchitecture. Biomaterials. 2012;33:4157-65. 
[37] Wood GC, Keech MK. The formation of fibrils from collagen solutions. 1. The effect of experimental conditions: kinetic and electron-microscope studies. Biochem J. 1960;75:588-98.

[38] Li Y, Asadi A, Monroe MR, Douglas EP. pH effects on collagen fibrillogenesis in vitro: Electrostatic interactions and phosphate binding. Materials Science and Engineering: C. 2009;29:1643-9.

[39] Charras G, Sahai E. Physical influences of the extracellular environment on cell migration. Nat Rev Mol Cell Biol. 2014;15:813-24.

[40] Roeder BA, Kokini K, Sturgis JE, Robinson JP, Voytik-Harbin SL. Tensile Mechanical Properties of ThreeDimensional Type I Collagen Extracellular Matrices With Varied Microstructure. Journal of Biomechanical Engineering. 2002;124:214.

[41] Mertz EL, Leikin S. Interactions of inorganic phosphate and sulfate anions with collagen. Biochemistry. 2004;43:14901-12.

[42] Kurniawan NA, Enemark S, Rajagopalan R. The role of structure in the nonlinear mechanics of cross-linked semiflexible polymer networks. J Chem Phys. 2012;136:065101.

[43] Vargo-Gogola T, Rosen JM. Modelling breast cancer: one size does not fit all. Nat Rev Cancer. 2007;7:65972.

[44] Holliday DL, Speirs V. Choosing the right cell line for breast cancer research. Breast Cancer Res. 2011;13:215.

[45] Geiger B, Bershadsky A. Assembly and mechanosensory function of focal contacts. Current Opinion in Cell Biology. 2001;13:584-92.

[46] Friedl P. Prespecification and plasticity: shifting mechanisms of cell migration. Curr Opin Cell Biol. 2004;16:14-23.

[47] Weigelin B, Bakker G-J, Friedl P. Intravital third harmonic generation microscopy of collective melanoma cell invasion. IntraVital. 2014;1:32-43.

[48] Provenzano PP, Inman DR, Eliceiri KW, Trier SM, Keely PJ. Contact guidance mediated three-dimensional cell migration is regulated by Rho/ROCK-dependent matrix reorganization. Biophys J. 2008;95:5374-84.

[49] Zaman MH, Trapani LM, Sieminski AL, Mackellar D, Gong H, Kamm RD, et al. Migration of tumor cells in $3 \mathrm{D}$ matrices is governed by matrix stiffness along with cell-matrix adhesion and proteolysis. Proc Natl Acad Sci U S A. 2006;103:10889-94.

[50] Ehrbar M, Sala A, Lienemann P, Ranga A, Mosiewicz K, Bittermann A, et al. Elucidating the role of matrix stiffness in 3D cell migration and remodeling. Biophys J. 2011;100:284-93. 